\title{
Genuine empirical pressure within the proton
}

\author{
Adam Freese $\odot^{*}$ and Gerald A. Miller ${ }^{\dagger}$ \\ Department of Physics, University of Washington, Seattle, Washington 98195, USA
}

(Received 22 April 2021; accepted 8 July 2021; published 30 July 2021)

\begin{abstract}
A phenomenological extraction of forces within the proton has recently been performed using JLab CLAS data V. D. Burkert et al., [arXiv:2104.02031]. The extraction used a three-dimensional Breit frame description in which the initial and final proton states have different momenta. Instead, we obtain the twodimensional (2D) transverse light front pressure densities that incorporate relativistic effects arising from the boosts that cause the initial and final states to differ. Taking the CLAS results seriously, we obtain a 2D mechanical radius for the proton. Additionally, we find predominantly repulsive forces near the proton's center, and predominantly attractive forces further out.
\end{abstract}

DOI: 10.1103/PhysRevD.104.014024

\section{INTRODUCTION}

The goal of determining the magnitude and spatial distribution of forces within hadrons has garnered great recent interest [1-8]. Information about internal forces within hadrons is encoded in the energy-momentum tensor (EMT) $[1,9,10]$, which additionally contains information about the decomposition and distribution of energy via a form factor, $A(t)$ [11-16] and angular momentum via a form factor, $J(t)$ [17-19]. The variable $t$ represents the square of the momentum transfer between initial and final proton states. The focus here is the third form factor, $D(t)$ [1], which encodes information about internal forces. The three form factors represent separately conserved contributions to the EMT.

Recently, data from Jefferson Lab have been used to infer $D(t)$ and the pressure distribution within the proton $[3,20]$. The obtained three-dimensional pressure distribution does not incorporate relativistic effects caused by boosts that must be incorporated when $t R^{2} \sim 1$, where $R$ is a measure of the size of the system. Obtaining spatial distributions requires an integral over all values of $t$, so determining the proton's internal structure requires a fully relativistic approach.

The relativistic effects due to boosts can be incorporated into spatial densities by using light front coordinates and defining the density at fixed light front time [10,21-24]. This can be done because the Poincaré group has a

\footnotetext{
*afreese@uw.edu

miller@uw.edu
}

Published by the American Physical Society under the terms of the Creative Commons Attribution 4.0 International license. Further distribution of this work must maintain attribution to the author(s) and the published article's title, journal citation, and DOI. Funded by SCOAP ${ }^{3}$.
Galilean subgroup that commutes with the light front Hamiltonian [25-27]. The densities obtained in this way involve integrating out a spatial coordinate in the light front direction, giving a two-dimensional (2D) density on the transverse plane. The formalism for using light front coordinates to obtain a relativistically correct pressure density was explicated in Ref. [10]. Thus we use the light front formalism to obtain a relativistically correct pressure density from the Jefferson Lab $D(t)$ extraction.

\section{LIGHT FRONT FORMALISM}

In the light front formalism, spacetime is parametrized in terms of coordinates $\left(x^{+}, x^{-}, \mathbf{x}_{\perp}\right)$, where $x^{ \pm}=$ $\frac{1}{\sqrt{2}}\left(x^{0} \pm x^{3}\right) \cdot x^{+}$is considered the "time" variable. For transverse densities in particular, all dependence on $x^{-}$is integrated out, giving a $(2+1)$-dimensional picture in terms of the transverse spatial coordinates $\mathbf{x}_{\perp}$. Within this $(2+1)$-dimensional picture, the EMT-when sandwiched within physical state kets-can be written as

$$
\left\langle\Psi\left|T_{\mathrm{LF}}^{\mu \nu}(x)\right| \Psi\right\rangle=u^{\mu}(x) u^{\nu}(x) \varepsilon(x)+S^{\mu \nu}(x) .
$$

Here, $x=\left(x^{+}, \mathbf{x}_{\perp}\right)$, and $\mu$ and $\nu$ range only over $+, 1,2$. The wave-packet state $|\Psi\rangle$ is a superposition of momentum eigenstates such that the transverse position is well defined. The variable $\varepsilon(x)$ is the $P^{+}$(light front momentum) density, and $u^{\mu}(x)$ encodes the flow of the hadron-which includes not just motion of the quarks and gluons within it, but also movement of the wave packet due to dispersion. The tensor $S^{\mu \nu}(x)$ is the "pure stress tensor," and corresponds to the spatial components of the EMT as measured by a locally comoving observer. [i.e., an observer who sees $u^{\mu}(x)=0$ at their current location]. It is the pure stress tensor that encodes the distribution of pressure and shear forces in the hadron. 
For a transversely localized state with definite light front helicity (i.e., polarized in the $\hat{z}$ direction), the pure stress tensor is related to $D(t)$ by a two-dimensional Fourier transform:

$$
\begin{aligned}
S^{i j}\left(\mathbf{x}_{\perp}\right)= & \frac{1}{4 P^{+}} \int \frac{\mathrm{d}^{2} \boldsymbol{\Delta}_{\perp}}{(2 \pi)^{2}}\left(\boldsymbol{\Delta}_{\perp}^{i} \Delta_{\perp}^{j}-\boldsymbol{\Delta}_{\perp}^{2} \delta^{i j}\right) \\
& D\left(-\boldsymbol{\Delta}_{\perp}^{2}\right) e^{-i \boldsymbol{\Delta}_{\perp} \cdot \mathbf{x}_{\perp}} .
\end{aligned}
$$

It can be decomposed into an isotropic pressure function $p\left(\mathbf{x}_{\perp}\right)$ and a shear stress function $s\left(\mathbf{x}_{\perp}\right)$ :

$$
S^{i j}\left(\mathbf{x}_{\perp}\right)=\delta^{i j} p\left(\mathbf{x}_{\perp}\right)+\left(\frac{x_{\perp}^{i} x_{\perp}^{j}}{x_{\perp}^{2}}-\frac{1}{2} \delta^{i j}\right) s\left(\mathbf{x}_{\perp}\right) .
$$

Since these are two-dimensional transverse quantities, the pressure has units of force/length instead of force/area. The pure stress tensor also gives the expected net force density acting at any point $\mathbf{x}_{\perp}$ within the hadron via

$$
\mathbf{F}_{\perp}^{j}\left(\mathbf{x}_{\perp}\right)=-\nabla_{i} S^{i j}\left(\mathbf{x}_{\perp}\right) .
$$

For an equilibrium system such as an isolated hadron,

$$
\mathbf{F}_{\perp}\left(\mathbf{x}_{\perp}\right)=0
$$

identically. This force-balance condition can be seen to follow from Eq. (2). Since these densities are defined through expectation values, all pressures and forces should likewise be interpreted as expectation values.

\section{A. Radial and tangential pressures}

Although the net force everywhere in the hadron is zero, there is nonetheless a static anisotropic pressure that would be felt by a hypothetical pressure gauge immersed within the hadron. $S^{i j}\left(\mathbf{x}_{\perp}\right)$ in particular encodes such pressures as measured by a gauge that is comoving along with the hadron flow encoded in $u^{\mu}\left(\mathbf{x}_{\perp}\right)$. The force that would be measured by such a gauge is given by

$$
\mathbf{F}_{\text {gauge }}^{j}=\int_{L} \mathrm{~d} l \hat{u}_{i} S^{i j}\left(\mathbf{x}_{\perp}\right),
$$

where $L$ is the one-dimensional surface of the gauge and $\hat{u}_{i}$ is an inward-facing unit normal vector to that surface.

By appropriately considering gauges in different orientations, one can obtain expressions for the radial and tangential pressure within a hadron:

$$
\begin{aligned}
& p_{r}\left(\mathbf{x}_{\perp}\right)=\hat{r}_{i} \hat{r}_{j} S^{i j}\left(\mathbf{x}_{\perp}\right)=p\left(\mathbf{x}_{\perp}\right)+\frac{1}{2} s\left(\mathbf{x}_{\perp}\right), \\
& p_{t}\left(\mathbf{x}_{\perp}\right)=\hat{\phi}_{i} \hat{\phi}_{j} S^{i j}\left(\mathbf{x}_{\perp}\right)=p\left(\mathbf{x}_{\perp}\right)-\frac{1}{2} s\left(\mathbf{x}_{\perp}\right) .
\end{aligned}
$$

We follow Ref. [28] in calling these "pressures." References [1,10] refer to $p_{r}\left(\mathbf{x}_{\perp}\right)$ (or its Breit frame analogue) as a "normal force," but we avoid such nomenclature here in order to maintain clarity that the net force everywhere in the hadron is zero. References [1,10,28,29] postulate $p_{r}\left(x_{\perp}\right) \geq 0$ as a stability condition, but there are no sign constraints on $p_{t}\left(x_{\perp}\right)$.

Since $p_{r}\left(\mathbf{x}_{\perp}\right)$ is strictly non-negative, it can be used to define a mechanical radius $[1,10]$, which comes out as

$$
\left\langle x_{\perp}^{2}(\text { mech })\right\rangle=\frac{\int \mathrm{d}^{2} \mathbf{x}_{\perp} \mathbf{x}_{\perp}^{2} p_{r}\left(\mathbf{x}_{\perp}\right)}{\int \mathrm{d}^{2} \mathbf{x}_{\perp} p_{r}\left(\mathbf{x}_{\perp}\right)}=\frac{4 D(0)}{\int_{-\infty}^{0} \mathrm{~d} t D(t)} .
$$

This means that determining the mechanical radius requires knowing $D(t)$ for both small and large values of $-t$.

\section{EMPIRICAL TRANSVERSE PRESSURES}

The form factor $D(t)$ can in principle be extracted directly from the Compton form factor $\mathcal{H}(\xi, t)$ using dispersion relations [3,30-32]. This was done in Ref. [3] through a dispersive analysis of deeply virtual Compton scattering (DVCS) data from CLAS at Jefferson Lab $[3,33,34]$.

A major caveat attached to the extraction is that it includes only quarks, since gluons do not contribute to DVCS at leading order. Thus any pressure densities presented in this work are just the quark contributions to these pressures. Moreover, since gluons are not being included, there is in principle an additional form factor $\bar{c}_{q}(t)$ that can contribute to the isotropic pressure density [1]. However, phenomenological and model estimates $[14,28,35]$ have $\left|\bar{c}_{q}(0)\right| \ll\left|D_{q}(0)\right|$, so we neglect the contributions of this largely unknown form factor.

An additional major caveat is that $D(t)$ is only the first term in a series expansion of the subtraction term in the DVCS dispersion relations, and keeping only this first term may result in large systematic uncertainties; see Ref. [36] for a discussion of this issue.

With these caveats in mind, the authors of Ref. [20] have fit $D(t)$ to the following functional form:

$$
D(t)=\frac{D(0)}{\left(1-t / \Lambda^{2}\right)^{\alpha}},
$$

where $D(0), \Lambda^{2}$, and $\alpha$ are the fit parameters. The values obtained by the authors of Ref. [20] are given in Table I.

Using the empirical fit parameters for $D(t)$, as well as the formalism explicated above, we obtain empirical estimates for the isotropic, radial, and tangential pressure of the

TABLE I. Parameters for Eq. (10) from Ref. [20], including fit and systematic errors. It should be noted that the systematic errors include systematics of assuming a simple multipole form for $D(t)$; see the discussion in Ref. [20].

\begin{tabular}{lcc}
\hline \hline$D(0)$ & $\Lambda^{2}\left(\mathrm{GeV}^{2}\right)$ & $\alpha$ \\
\hline$-1.47 \pm 0.06 \pm 0.14$ & $1.02 \pm 0.13 \pm 0.21$ & $2.76 \pm 0.23 \pm 0.48$ \\
\hline \hline
\end{tabular}




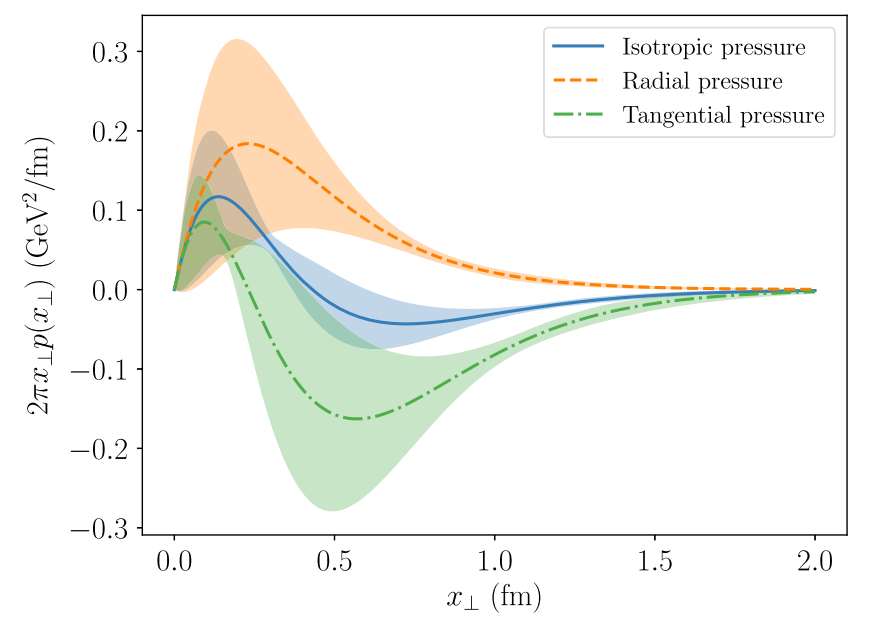

FIG. 1. The isotropic, radial, and tangential pressures within the proton on the light front, as suggested by the parameters in Table I. A state- and frame-dependent factor $1 / P^{+}$has been removed from the plotted quantities. The error band includes only fit uncertainty.

proton in a definite light front helicity state. For such a state, these pressures are functions of only the magnitude $x_{\perp}$ of $\mathbf{x}_{\perp}$. These quantities, weighted by $2 \pi x_{\perp}$, are given in Fig. 1. A factor of $1 / P^{+}$was removed from the plotted quantities, making the plotted quantities state- and frameindependent.

The quantity $P^{+} p\left(x_{\perp}\right)$ can also be cast into units of pascals. However, the numbers obtained should not be interpreted as literal forces per unit area, since-by integrating out $x^{-}$- the light front formalism is inherently $(2+1)$-dimensional. Nonetheless, $P^{+} p\left(x_{\perp}\right)$ is a state- and frame-independent quantity that can be cast into units of pascals, and accordingly encodes some intrinsic property of the proton, and may also give some intuitive insight into the rough magnitude of pressures present inside the proton. We find with the parameters from Ref. [20] that $P^{+} p(0) \approx 2.36 \times 10^{35} \mathrm{~Pa}$ - the same order of magnitude suggested by the Breit frame analysis of Ref. [3].

Using the form in Eq. (10), there is a simple expression for the mechanical radius, or pressure radius:

$$
\left\langle x_{\perp}^{2}(\text { mech })\right\rangle=\frac{4(\alpha-1)}{\Lambda^{2}} .
$$

Our result for the pressure radius is given in Table II, which also includes several other light front proton radii. Note that light front radii differ from the usual three-dimensional radii defined in the literature, and are usually just a factor $\sqrt{2 / 3}$ smaller. The charge radius, obtained from the Dirac form factor $F_{1}(\mathrm{t})$, also differs from the usual Sachs radius due to relativistic spin effects [22]:

$$
\left\langle x_{\perp}^{2}(\text { charge })\right\rangle=\frac{2}{3} r_{\text {Sachs }}^{2}-\frac{\kappa}{M},
$$

where $\kappa$ is the proton's anomalous magnetic moment.
Looking at Table II, the systematic error bars on the pressure radius make any definitive comparison between it and the other radii difficult. However, taking the central values seriously yields an approximate ordering of the rootmean-square radii:

$x_{\perp}($ mass $) \lesssim x_{\perp}($ pressure $) \lesssim x_{\perp}($ axial $) \lesssim x_{\perp}($ charge $)$

Crucially, the apparent spatial extent of the proton differs depending on how its spatial extent is defined-and the proton can look bigger or smaller depending on what probe or process is used. Taking these as strict inequalities cannot be justified with the uncertainties quoted in Table II. If this ordering roughly holds, it is worth speculating on what factors might be at play.

To start, the charge radius notoriously obtains a contribution from the pion cloud [40-42] that is absent from the axial radius [43], the latter of which is expected to be smaller for this reason. By contrast, the pion cloud does carry energy and can reasonably be expected to exert pressure, and thus we may expect it to contribute to the mass and pressure radii.

Other factors are likely at play, however. The "mass radius" is actually the radius of the $P^{+}$density [5,10], and accordingly weighs configurations more strongly when a single quark carries a large portion of the proton's forward momentum. These configurations notoriously have small spatial extent $[44,45]$, thus biasing the mass radius towards being small. The pressure radius may also tend towards being small because pressure compounds upon itself at greater "depth," i.e., closer to the proton center. It would be interesting to know with greater certainty whether the pressure radius really exceeds the mass radius, and also how it compares to the axial radius. It would thus be prudent to pursue higher-precision measurements of DVCS from the proton - as well as to pursue methods to control and reduce systematic uncertainty in the extraction of $D(t)$-in order to obtain stronger constraints on the proton pressure densities and its mechanical radius.

TABLE II. Approximate root-mean-square radii of the proton on the light front. The quoted sources provide a three-dimensional radius that we have converted into a $2 \mathrm{D}$ light front radius

\begin{tabular}{|c|c|c|c|c|}
\hline & Radius & Uncertainty (fm) & Definition & Source \\
\hline & 0.518 & $62+0$ & $\mathrm{E}$ & Thi \\
\hline las & 0.4 & 0.02 & $\sqrt{4 A^{\prime}(0)}$ & Ref. [37] \\
\hline xial & 0.55 & 0.17 & $\sqrt{4 G_{A}^{\prime}(0)}$ & Ref. [38] \\
\hline harge & 0.6266 & 0.0017 & $\sqrt{4 F_{1}^{\prime}(0)}$ & Ref. [39] \\
\hline
\end{tabular}
using the definitions given in the table.

${ }^{\text {a}}$ The mass radius extracted from Ref. [37] should be taken as preliminary and subject to future corrections. The exact relationship between the scalar form factor $G(t)$ and the mass form factor remains to be elucidated, and likewise for the theoretical foundations of meson dominance in graviton interactions. 


\section{A. Effects of polarization}

It is possible to obtain pressure densities for transversely polarized protons within the light front formalism. Transverse polarization states are given by superpositions of light front helicity states,

$$
\left|s_{T}=\mathbf{s}_{\perp}\right\rangle=\frac{\left|\lambda=+\frac{1}{2}\right\rangle+e^{i \phi_{s}}\left|\lambda=-\frac{1}{2}\right\rangle}{\sqrt{2}},
$$

and accordingly, expectation values for transverse polarization states involve helicity-flip matrix elements.

Because of these helicity-flip terms, proton densitiesincluding the pressure densities - obtain a dependence on the angle $\phi=\phi_{x}-\phi_{s}$ between $\mathbf{x}_{\perp}$ and $\mathbf{s}_{\perp}$. If we use $p\left(x_{\perp}\right)$ to denote the pressure density of a light front helicity state, the pressure density for a transversely polarized state is
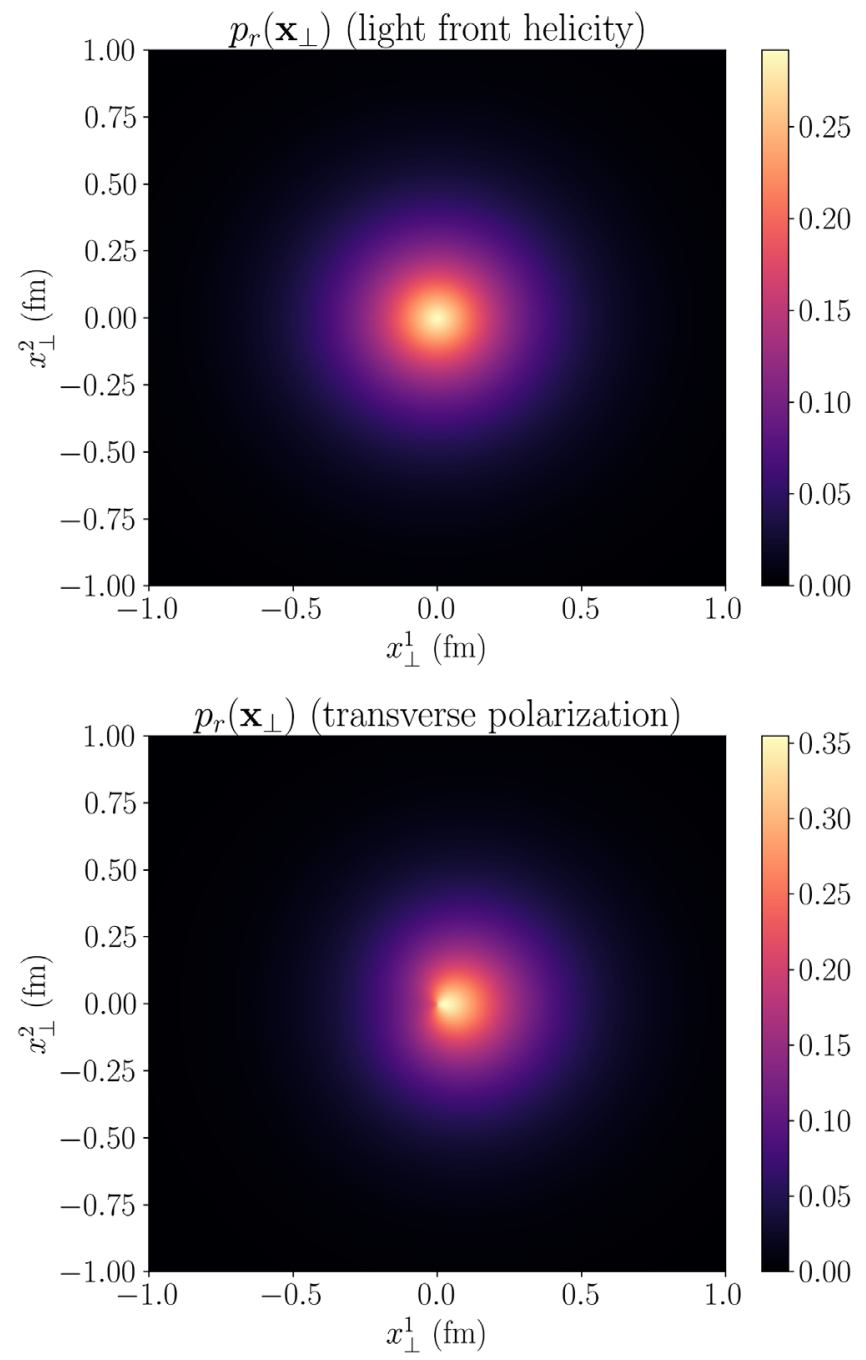

FIG. 2. 2D plots of the radially directed pressure in the proton. A factor $1 / P^{+}$has been removed from the plotted quantities, which are in units of $\mathrm{GeV}^{2} / \mathrm{fm}^{2}$. The top panel is for longitudinally polarized protons, and the bottom panel for transversely polarized protons with the spin in the $+\hat{y}$ direction.

$$
p_{T}\left(x_{\perp}, \phi\right)=p\left(x_{\perp}\right)+\frac{\sin \phi}{2 M} p^{\prime}\left(x_{\perp}\right) .
$$

This relation applies to all of the isotropic, radial, and tangential pressures.

The 2D radial pressure densities for longitudinally and transversely polarized protons are plotted in Fig. 2. The longitudinally polarized proton has an azimuthally symmetric pressure. However, the transversely polarized proton has a greater concentration of pressure to the right of $\left(-90^{\circ}\right.$ from) the spin direction. This finding is reminiscent of a similar finding about electric charge density in Ref. [46].

Interestingly, the transverse pressure distribution suggests that - when analyzed in a light front framework using pressure densities - the proton is not shaped like a sphere. This is not too surprising, since the spin axis identifies a particular direction in space, with respect to which directions such as right and left can be defined [47].

\section{DISCUSSION AND INTERPRETATION}

When interpreting the results for the pressures, it is important to keep in mind their proper physical interpretations. First of all, since densities are defined via expectation values of operators for a physical proton state, the forces and pressures obtained by this formalism are all expectation values (in the usual quantum-mechanical sense), rather than exact values as in a classical continuum system. Another especially important fact to bear in mind is that the (expected) net force everywhere in the hadron is identically zero-a statement that the hadron is in internal equilibrium, as is expected of a system in its ground state.

We clarify the situation further using Fig. 3, which depicts the forces exerted on a small slab within the proton by the remainder of the proton, specifically in a case where $p_{r}>0$ and $p_{t}<0$. The net force on this piece of the proton is zero, but there are nonzero forces acting on each side of the slab. Since $p_{r}\left(x_{\perp}\right) \geq 0$, the radially facing sides of

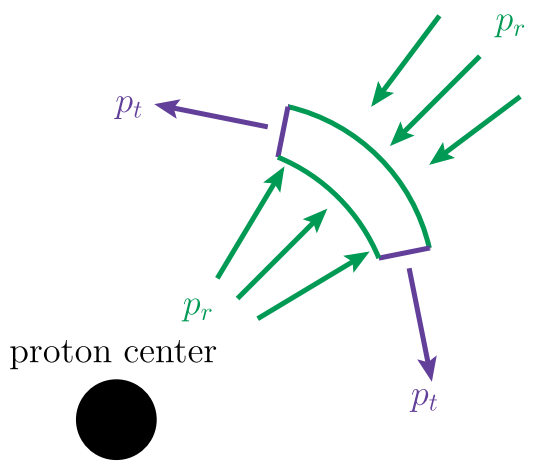

FIG. 3. A cartoon depicting (expectation values of) forces acting on a slab within the proton. The net force acting on this slab is zero, but forces acting on any side of the slab from the outside may be nonzero. These nonzero surface forces constitute the pressure in the proton. 
the slab are both pushed on from the outside. When $p_{t}\left(x_{\perp}\right)>0$, the tangentially facing sides are also pushed on, but when $p_{t}\left(x_{\perp}\right)<0$, these sides are pulled on by the remainder of the proton instead.

As seen in Fig. 1, the radial pressure in the proton is strictly positive. Although attractive and repulsive forces are both present in the proton, the repulsive forces overwhelm the attractive forces in the radial direction at all distances. The balance of forces keeping the proton in equilibrium is thus primarily, in the radial direction, between repulsive forces acting in both the inward and outward radial directions.

On the other hand, somewhere between around 0.12$0.34 \mathrm{fm}$ from the proton's center, the tangential pressure changes sign from positive to negative. This means that at distances less than this, the forces in all directions are primarily repulsive, while at greater distances, the forces in the $\pm \hat{\phi}$ directions are primarily attractive. This leads to a scenario where elements of the proton that are far from its center are being pushed from the radial directions and pulled around the proton, suggesting a reverse spaghettification.

The isotropic pressure $p\left(x_{\perp}\right)$ averages over the pressures in all directions, telling us on average whether the majority of forces at a distance $x_{\perp}$ from the proton's center are repulsive or attractive. The isotropic pressure crosses zero somewhere from around $0.31-0.55 \mathrm{fm}$, meaning the forces at shorter distances are primarily repulsive and forces at longer distances are primarily attractive. We stress, however, that the forces at these spatial locations are primarily repulsive or attractive averaged over directions, and not towards or away from the proton's center.

The exact numbers are subject to correction as stronger empirical constraints on the systematics of extracting $D(t)$ are achieved. However, the qualitative picture described above is expected to hold.
Another especially important caveat to bear in mind while applying concepts of force and pressure to the proton is that utilizing these concepts does not imply the proton to behave like a fluid, nor like any other particular macroscopic continuum system. The stress tensor and pressure have applications to a variety of both liquid and solid continuum systems [48-51]. As an object much smaller than and drastically different from macroscopic systems in solid or fluid states, the proton should not be expected to behave either like a viscous fluid nor like an elastic solid; for instance, one should not expect quarks in the proton to obey a Navier-Stokes equation. The stress tensor, and the resultant forces and pressures, can be formally defined for any continuum system.

\section{CONCLUSION}

The empirical extraction of $D(t)$ in Ref. [20] was used to obtain transverse densities of the isotropic, radial, and tangential pressures in the proton within the light front formalism. A physical interpretation of these pressures was provided along with computations of the empirical mechanical radius associated with them. Since-in contrast to the picture provided by the Breit frame-transverse densities on the light front are relativistically correct, the densities obtained in this work should be interpreted as the genuine empirical pressure densities of the proton implied by the findings of Ref. [20].

\section{ACKNOWLEDGMENTS}

The authors would like to thank Volker Burkert, Latifa Elouadrhiri, F. X. Girod and M. V. Polyakov for helpful correspondence. This work was supported by the U.S. Department of Energy Office of Science, Office of Nuclear Physics under Award No. DE-FG02-97ER-41014.
[1] M. V. Polyakov and P. Schweitzer, Int. J. Mod. Phys. A 33, 1830025 (2018).

[2] P. E. Shanahan and W. Detmold, Phys. Rev. Lett. 122, 072003 (2019).

[3] V.D. Burkert, L. Elouadrhiri, and F.X. Girod, Nature (London) 557, 396 (2018).

[4] K. Kumerički, Nature (London) 570, E1 (2019).

[5] A. Freese and I. C. Cloët, Phys. Rev. C 100, 015201 (2019).

[6] I. V. Anikin, Particles 2, 357 (2019).

[7] M. J. Neubelt, A. Sampino, J. Hudson, K. Tezgin, and P. Schweitzer, Phys. Rev. D 101, 034013 (2020).

[8] M. Varma and P. Schweitzer, Phys. Rev. D 102, 014047 (2020).

[9] M. V. Polyakov, Phys. Lett. B 555, 57 (2003).
[10] A. Freese and G. A. Miller, Phys. Rev. D 103, 094023 (2021).

[11] X.-D. Ji, Phys. Rev. Lett. 74, 1071 (1995).

[12] X.-D. Ji, Phys. Rev. D 52, 271 (1995).

[13] C. Lorcé, Eur. Phys. J. C 78, 120 (2018).

[14] Y. Hatta, A. Rajan, and K. Tanaka, J. High Energy Phys. 12 (2018) 008.

[15] S. Rodini, A. Metz, and B. Pasquini, J. High Energy Phys. 09 (2020) 067.

[16] A. Metz, B. Pasquini, and S. Rodini, Phys. Rev. D 102, 114042 (2020).

[17] J. Ashman et al. (European Muon Collaboration), Phys. Lett. B 206, 364 (1988).

[18] X.-D. Ji, Phys. Rev. Lett. 78, 610 (1997). 
[19] E. Leader and C. Lorcé, Phys. Rep. 541, 163 (2014).

[20] V. D. Burkert, L. Elouadrhiri, and F.X. Girod, arXiv: 2104.02031.

[21] M. Burkardt, Int. J. Mod. Phys. A 18, 173 (2003).

[22] G. A. Miller, Phys. Rev. Lett. 99, 112001 (2007).

[23] G. A. Miller, Phys. Rev. C 80, 045210 (2009).

[24] G. A. Miller, Phys. Rev. C 99, 035202 (2019).

[25] P. A. M. Dirac, Rev. Mod. Phys. 21, 392 (1949).

[26] L. Susskind, Phys. Rev. 165, 1535 (1968).

[27] S. J. Brodsky, H.-C. Pauli, and S. S. Pinsky, Phys. Rep. 301, 299 (1998).

[28] C. Lorcé, H. Moutarde, and A. P. Trawiński, Eur. Phys. J. C 79, 89 (2019).

[29] I. A. Perevalova, M. V. Polyakov, and P. Schweitzer, Phys. Rev. D 94, 054024 (2016).

[30] M. Diehl and D. Y. Ivanov, Eur. Phys. J. C 52, 919 (2007).

[31] I. V. Anikin and O. V. Teryaev, Fiz. B 17, 151 (2008).

[32] B. Pasquini, M. V. Polyakov, and M. Vanderhaeghen, Phys. Lett. B 739, 133 (2014).

[33] F. X. Girod et al. (CLAS Collaboration), Phys. Rev. Lett. 100, 162002 (2008).

[34] H. S. Jo et al. (CLAS Collaboration), Phys. Rev. Lett. 115, 212003 (2015).

[35] M. V. Polyakov and H.-D. Son, J. High Energy Phys. 09 (2018) 156.

[36] H. Dutrieux, C. Lorcé, H. Moutarde, P. Sznajder, A. Trawiński, and J. Wagner, Eur. Phys. J. C 81, 300 (2021).

[37] D. E. Kharzeev, arXiv:2102.00110.

[38] R. J. Hill, P. Kammel, W. J. Marciano, and A. Sirlin, Rep. Prog. Phys. 81, 096301 (2018).
[39] P. J. Mohr, B. N. Newell, D. B. Taylor, and E. Tiesinga, CODATA Recommended Values of the Fundamental Physical Constants: 2018 (2018).

[40] A. W. Thomas, S. Theberge, and G. A. Miller, Phys. Rev. D 24, 216 (1981).

[41] I. C. Cloet and G. A. Miller, Phys. Rev. C 86, 015208 (2012).

[42] I. C. Cloët, W. Bentz, and A. W. Thomas, Phys. Rev. C 90, 045202 (2014).

[43] M. Strikman and C. Weiss, Phys. Rev. D 80, 114029 (2009).

[44] L. L. Frankfurt and M. I. Strikman, Nucl. Phys. B250, 143 (1985).

[45] O. Hen, G. A. Miller, E. Piasetzky, and L. B. Weinstein, Rev. Mod. Phys. 89, 045002 (2017).

[46] C. E. Carlson and M. Vanderhaeghen, Phys. Rev. Lett. 100, 032004 (2008).

[47] G. A. Miller, Phys. Rev. C 68, 022201 (2003).

[48] A. Sommerfeld, Mechanics of Deformable Bodies: Lectures on Theoretical Physics, Vol. 2 (Elsevier Science, New York, 2016).

[49] A. L. Fetter and J. D. Walecka, Theoretical Mechanics of Particles and Continua, International Series in Pure and Applied Physics (McGraw-Hill, New York, NY, 1980).

[50] C. Batchelor and G. Batchelor, An Introduction to Fluid Dynamics, Cambridge Mathematical Library (Cambridge University Press Cambridge, England, 2000).

[51] F. Irgens, Continuum Mechanics (Springer, Berlin, Heidelberg, 2008). 